\title{
Subsidios lexicográficos para la enseñanza de lenguas extranjeras: Qué diccionarios tienen a su disposición los aprendices brasileños de español?
}

\section{Lexicographical resources for foreign language teaching: What dictionaries are available to Brazilian learners of Spanish?}

Virginia Sita Farias*

Universidade Federal do Rio Grande do Sul

Porto Alegre - Rio Grande do Sul / Brasil

RESUMEN: Este trabajo tiene dos objetivos. En primer lugar, presentar un panorama de los diccionarios de lengua española mediante la elaboración de una taxonomía. En segundo lugar, teniendo en cuenta las necesidades específicas de los aprendices brasileños de español, evaluar cuán realmente útiles pueden ser los diccionarios de lengua española para esos estudiantes, poniendo especial atención a los diccionarios bilingües, a los learner's dictionaries y a los diccionarios generales de lengua.

PALABRAS CLAVE: Lexicografía, diccionarios de español, aprendices brasileños de español.

ABSTRACT: This paper has two objectives. Firstly, we will present an overview of Spanish dictionaries through the elaboration of a taxonomy. Secondly, taking into account the specific language needs of Brazilian learners of Spanish, we will evaluate how helpful the Spanish dictionaries are for the students, paying special attention to bilingual dictionaries, learner's dictionaries and general monolingual dictionaries.

KEYWORDS: Lexicography, Spanish dictionaries, Brazilian learners of Spanish.

\section{Introducción}

El diccionario es, por naturaleza, un instrumento didáctico. De acuerdo con Alvar Ezquerra (1993, apud HERNÁNDEZ, 1998, p. 49) "el diccionario

\footnotetext{
*virginiafarias@terra.com.br
} 
cumple con su misión didáctica desde el momento en que el usuario se acerca a él para ver cómo se escribe una palabra, qué significa, si puede ser utilizada en un sentido determinado, o en una construcción cualquiera, etc.”. Sin embargo, hace falta señalar que los diccionarios no son todos iguales, ni tampoco aspiran a lograr los mismos objetivos (cf. HERNÁNDEZ, 1998, p. 49). En términos bien simples, un usuario que desea traducir una determinada palabra de una lengua extranjera a su lengua materna, a lo mejor, consultará un diccionario bilingüe. Por otra parte, si el mismo usuario no desea encontrar un equivalente en su lengua materna para una palabra de la lengua extranjera, sino más bien producir un texto en la lengua extranjera, lo ideal sería utilizar un diccionario específico para este fín. ${ }^{1}$

Teniendo en cuenta lo expuesto en el párrafo anterior, creemos que, para asegurar la calidad de una consulta al diccionario, es necesario definir a priori dos cosas: (a) quién es el usuario del diccionario y (b) cuál es la finalidad de la consulta. Estos dos parámetros guardan estrecha relación con los dos problemas fundamentales que la elaboración de un diccionario plantea: (a) la definición del perfil del usuario y (b) la definición tipológica del diccionario. ${ }^{2}$

La delimitación del usuario y de la finalidad de la consulta son factores determinantes para la clasificación tipológica de un diccionario (en especial, si estamos manejando una tipología basada en criterios funcionales), y pueden servir, a la vez, como criterios para la construcción del perfil del usuario. ${ }^{3} \mathrm{De}$ hecho, pues, los parámetros mencionados le sirven tanto al usuario que escoje el diccionario que mejor atiende sus necesidades de consulta, como al lexicógrafo que elabora una obra lexicográfica.

\footnotetext{
${ }^{1}$ Citamos como mejor ejemplo de este tipo de obra el LEA (2006), para aprendices de la lengua inglesa. Este diccionario presenta en cada artículo léxico un apartado que se denomina "related words" y se destina a la presentación de sinónimos, antónimos y otras palabras pertenecientes al mismo universo semántico del signo-lema.

${ }^{2}$ La relación entre la definición tipológica del diccionario y la finalidad de la consulta queda más en evidencia cuando se toma como parámetro una clasificación basada en criterios funcionales, o sea, en el uso efectivo del diccionario, a ejemplo de las clasificaciones propuestas por Kühn (1989), Hausmann (1989) y Engelberg y Lemnitzer (2004).

${ }^{3}$ Sirvan de ejemplo los parámetros propuestos en Bugueño y Farias (2008) para la elaboración de un perfil de usuario de un diccionario escolar de lengua portuguesa: (a) el nivel escolar al cual está dirigida la obra y (b) las habilidades lingüísticas que los estudiantes deben desarrollar en este período.
} 
En esta exposición pondremos nuestra atención en la perspectiva de los usuarios, teniendo en cuenta especialmente a los aprendices brasileños de español. Nuestro objetivo es ofrecer un panorama de las obras lexicográficas disponibles para el aprendiz brasileño de español, así como evaluar cuán realmente útiles pueden ser para un usuario cuya lengua materna es el portugués. Para que sea posible sacarle el mejor provecho a los diccionarios, se hace necesario que los aprendices tengan bien en claro tres aspectos extremadamente importantes:

1) cuáles son sus necesidades específicas en cada momento de su aprendizaje;

2) para qué y a quiénes sirve cada tipo específico de diccionario; y

3) qué problemas o limitaciones pueden presentar los diccionarios o cuáles son las dificultades que se pueden encontrar en su consulta.

\section{Breve panorama de los diccionarios de lengua española desde una perspectiva taxonómica}

Una taxonomía es un sistema de clasificación y descripción de elementos de una determinada categoría (cf. SWANEPOEL, 2003, p. 45). En el ámbito de los estudios lexicográficos se han desarrollado varias propuestas de clasificación de los diccionarios (cf., por ejemplo, ZGUSTA, 1971, p. 197 221; HAENSCH et al., 1982, p. 126-186; MARTÍNEZ DE SOUZA, 1995, s.v. diccionario; HARTMANN; JAMES, 2001, s.v. typology; LANDAU, 2001, p. 6-42; SWANEPOEL, 2003; ENGELBERG; LEMNITZER, 2004, p. 18-21). Sin embargo, según Bugueño (2003), hasta hoy no se ha elaborado una taxonomía perfecta y a la vez exhaustiva de obras lexicográficas. Esto se puede explicar por el hecho de que el problema de la clasificación de obras lexicográficas es hasta cierto punto insoluble. Kühn (1989), a su vez, ya planteaba que las taxonomías de diccionarios, en realidad, no son ni completas ni rigurosamente distintivas, debido a dos razones esenciales. En primer lugar, porque la aplicación de criterios distintos puede resultar en la descripción de genotipos idénticos. En segundo lugar, porque cada día surgen nuevos tipos de diccionarios.

Con el objetivo de presentar un breve panorama de las obras lexicográficas de lengua española, adoptaremos en este trabajo la taxonomía propuesta en Bugueño (2008) para la clasificación de los diccionarios alemanes. Hace falta señalar, sin embargo, que no todos los genotipos encontrados en la tradición lexicográfica alemana son igualmente encontrados 
en la tradición hispánica. Así, por ejemplo, hay genotipos como el diccionario ortográfico y el diccionario sintagmático que son muy representativos de la lexicografía alemana, pero que no tienen correspondientes en la lexicografía de lengua española. Por otra parte, la presencia masiva de los diccionarios de americanismos constituye un rasgo característico de la lexicografía hispánica. Estos hechos demuestran una vez más que Kühn (1989) tenía razón al afirmar que una taxonomía no puede ser universal. Adaptando, pues, la propuesta de Bugueño (2008) a las necesidades de la tradición lexicográfica de lengua española, tenemos la siguiente taxonomía: ${ }^{4}$

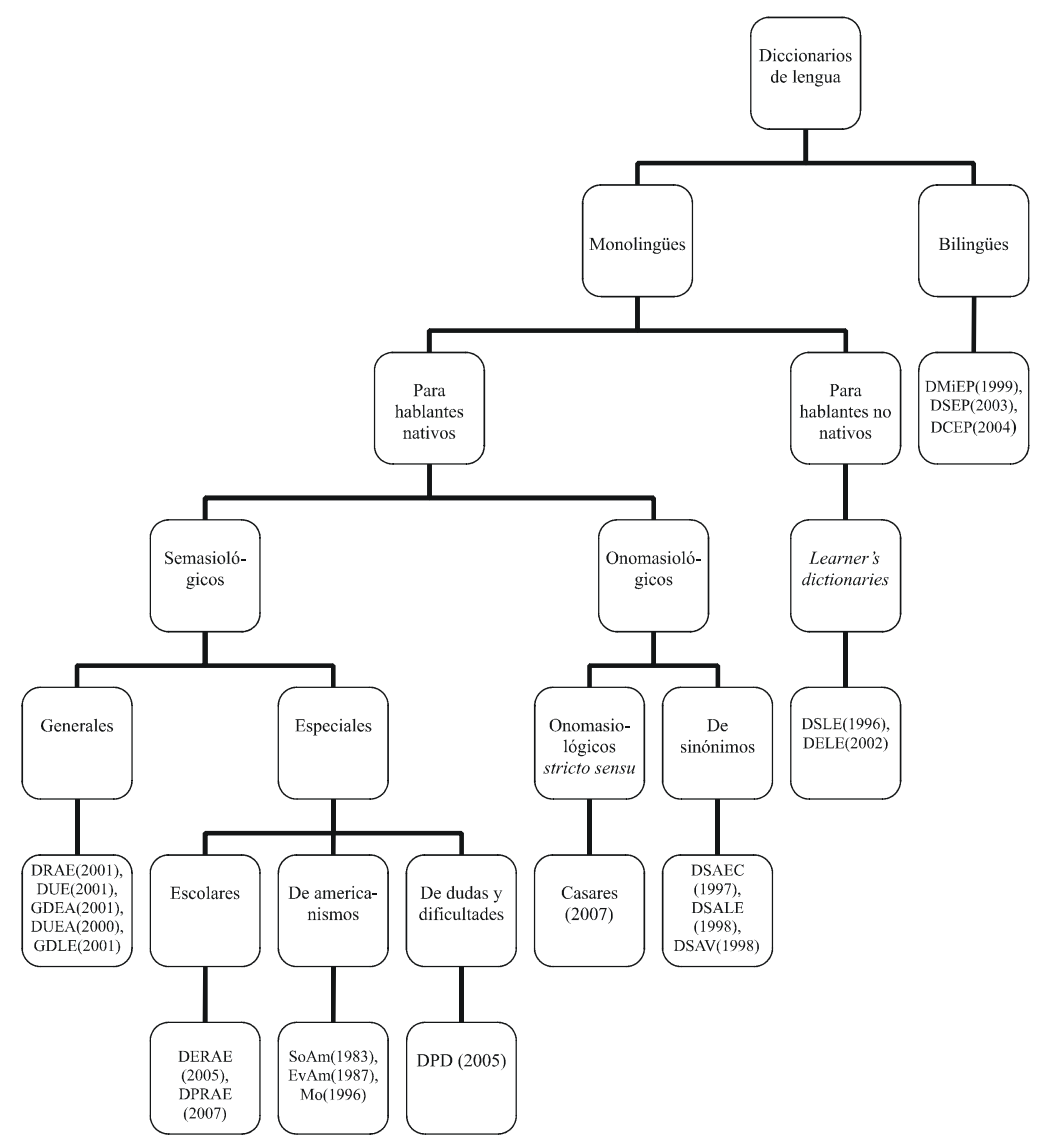

\footnotetext{
${ }^{4}$ Siguiendo la tendencia de la metalexicografía europea, se emplearán siglas para identificar las obras lexicográficas analizadas (cf. HARTMANN, 2001, p. 11).
} 
La primera distinción está basada en el número de lenguas. Así, pues, distinguimos entre los diccionarios monolingües y bilingües. En el ámbito de los diccionarios monolingües, se establece una separación entre diccionarios para hablantes nativos y para hablantes no nativos. Entre los diccionarios para hablantes nativos es posible distinguir entre semasiológicos y onomasiológicos. Respecto a los diccionarios semasiológicos, lo primeiro que hay que destacar es que ahí se establece una oposición funcional entre los diccionarios (cf. nota 2). De esta manera, tenemos de un lado los diccionarios generales (polifuncionales), y de otro, los diccionarios especiales (monofuncionales). ${ }^{5}$

Respecto a los diccionarios generales, el DRAE (2001) es, sin lugar a duda, la obra más prestigiada del mundo hispánico. A pesar de las muchas críticas que recibe (cf., por ejemplo, RABANALES, 1984), el diccionario de la Real Academia Española (RAE) es el parámetro para la fijación de la norma léxica, y en cierta medida, también lo es para la norma gramatical del español, además de constituir el modelo lexicográfico que han seguido los demás lexicógrafos (cf. ALVAR EZQUERRA, 1992, p. 644). También merece destacarse el DUE (2001). Aunque está destinado específicamente a los hablantes nativos del español, DUE (2001) es el diccionario monolingüe que mejor se puede ajustar a las necesidades de los aprendices de español como lengua extranjera que ya poseen un alto grado de proficiencia en esta lengua. Una de las contribuciones más importantes de su autora es la atención dedicada a los regímenes preposicionales y a las colocaciones en el diccionario (cf. HERNÁNDEZ, 1998, p. 73-74; ZANATTA, 2006a, p. 71-76). Por otra parte, subrayamos el hecho de que es muy difícil, para un usuario poco entrenado, consultar ese diccionario, entre otras cosas, en razón del elevado número de informaciones e íconos gráficos en el interior del artículo léxico. Señalamos aún que el DUE (2001) describe el español usual en España, de modo que, respecto al léxico, muchas palabras y acepciones que aparecen como desusadas en esa obra son americanismos, y respecto a la sintaxis, los ejemplos ofrecidos en general presentan el leísmo como norma, lo que, sumado al hecho de que los diccionarios del español suelen no marcar el dativo, puede dificultarle la vida al estudiante brasileño que necesita comprender la diferencia

\footnotetext{
${ }^{5}$ De acuerdo con lo expuesto en Kühn (1989), se definen como obras polifuncionales las que contienen un gran número de informaciones que le pueden interesar a un grupo más amplio de usuarios, en contraposición a las obras monofuncionales, que le sirven a las necesidades específicas de un grupo limitado de usuarios.
} 
entre los complementos directo e indirecto del español. Hay que mencionar todavía el GDEA (2001), que se destina a hablantes nativos y a aprendices extranjeros del español a la vez. Ese diccionario está basado en un corpus y pretende ser una descripción del español del final del siglo XX e inicio del siglo XXI, pero presenta algunas fallas ${ }^{6}$ que no le permiten cumplir su rol con perfección (cf. ZANATTA, 2006a, p. 57-63). En términos de diccionarios generales, merecen aún una mención DUEA (2000) y GDLE (2001).

En el ámbito de los diccionarios especiales, destacamos tres genotipos: el diccionario escolar, el diccionario de americanismos e el diccionario de dudas y dificultades. En lo concerniente a los diccionarios escolares, mencionamos el DERAE (2005) y el DPRAE (2007), ambos publicados por la RAE. En esta ocasión, no nos vamos a ocupar de esas obras. Destacamos, eso sí, que en realidad el panorama de la lexicografía pedagógica hispánica dedicada a hablantes nativos no es muy distinto al escenario brasileño. Las obras escolares hispánicas en general, igual que las brasileñas, ${ }^{7}$ suelen representar una continuidad de estilo y de forma respecto a los diccionarios generales monolingüies (cf. PÉREZ LAGOS, 1998, p. 115). Aunque algunos autores, como Pérez Lagos (1998), Hernández (1998) y Castillo y García (2003), ya apuntan hacia un cambio en este panorama, en realidad la "revolución" operada no es tan grande.

Como ya hemos dicho, en la lexicografía hispánica sobresale la presencia masiva de los llamados diccionarios de americanismos, tales como SoAm (1983), EvAm (1987) y Mo (1996). En Zanatta (2006b, p. 137), se define los americanismos como palabras o locuciones utilizadas específicamente en Hispanoamérica o que tienen en esta área un contenido semántico particular. Los diccionarios de americanismos, sin embargo, en su gran mayoría, no son obras de buena calidad: las marcas diatópicas, en general, no son confiables (cf. FORNARI, 2006), y las definiciones tampoco son de buena calidad (cf. ZANATTA, 2006b). Por fin, en lo que concierne a los diccionarios de

\footnotetext{
${ }^{6}$ Ponemos como ejemplo, en el plano macroestructural, las fallas en la selección de la nominata. Teniendo en cuenta que el GDEA (2001) es un diccionario que se propone presentar "los significados que los hablantes nativos de español asignan a las palabras en los inicios del siglo XXI" (GDEA, 2001, p. 7), no es consecuente registrar palabras marcadas como arcaicas, a ejemplo de murciégalo, paletó y pancera.

7 Para un panorama de los diccionarios escolares de lengua portuguesa, cf. Farias (2007) y Bugueño y Farias (2009).
} 
dudas y dificultades, mencionamos el DPD (2005), obra cuya publicación queda a cargo de la RAE. Bugueño (2006a) señala la importancia de este diccionario respecto a la fijación de la norma prescriptiva del español.

En la esfera de los diccionarios onomasiológicos, distinguimos entre onomasiológicos stricto sensu y de sinónimos. ${ }^{8}$ Entre los diccionarios onomasiológicos stricto sensu destacamos el ideológico de Casares (2007). Llamamos la atención sobre el hecho de que el gran problema de este tipo de obra es la organización del sistema conceptual, de manera que todavía no hay un consenso sobre cómo se debe generar la macroestructura de esos diccionarios (cf. MARTÍN MINGORANCE, 1994; PÉREZ LAGOS, 2001; BUGUEÑO, 2008). Así pues, a pesar de la importancia que se le atribuye a Casares (2007), su clasificación teocéntrica (Dios versus el Universo) tampoco escapa a la crítica (cf. PÉREZ LAGOS, 2001, p. 182).

Entre los diccionarios de sinónimos, mencionamos DSAEC (1997), DSALE (1998) y DSAV (1998). Salvo el DSAV (1998), que en algunos artículos presenta notas de uso que le sirven al usuario como distinguidores semánticos, las demás obras ofrecen solamente una serie de sinónimos y antónimos acumulativos.

De acuerdo con la separación esteblecida en la taxonomía propuesta, el segundo grupo de diccionarios monolingües comprende las obras destinadas a los hablantes no nativos, o sea, los llamados learner's dictionaries. Los dos únicos representantes de esa clase para el español son el DSLE (1996) y el DELE (2002), que es duramente criticado en Bugueño (2006b). En los apartados siguientes discutiremos los problemas de estas obras.

Llegamos finalmente a los diccionarios bilingües, que se oponen directamente a los diccionarios monolingües, en conformidad con la taxonomía presentada. Es posible encontrar una gran cantidad de obras bilingües que relacionan el español y el portugués. Sin embargo, la mayoría carecen de fundamentación teórico-metodológica adecuada, a ejemplo de las tres que mencionamos y que analizaremos más adelante.

\footnotetext{
${ }^{8}$ Los diccionarios bilingües también pueden ser considerados como onomasiológicos, teniendo en cuenta que la significación común entre el lema y el equivalente es un tertium comparationis entre las designaciones de las dos lenguas (cf. HARTMANN; JAMES, 2001, s.v. onomasiological dictionary).
} 


\section{Los estudiantes brasileños de español y los diccionarios de lengua española}

Teniendo en cuenta solamente a los aprendices de una lengua extranjera, es posible pensar en una suerte de ranking de obras de referencia utilizadas según el nivel de aprendizaje, el grado de proficiencia del estudiante, y, claro está, la tarea que se va a realizar. De esa manera, la primera opción siempre son los diccionarios bilingües. En segundo lugar, vienen los learner's dictionaries. Finalmente, en tercer lugar, están los diccionarios generales de lengua.

\section{Diccionarios bilingües}

La concepción y el diseño de un diccionario bilingüe implica que se consideren por lo menos cuatro variables: (a) las lenguas, (b) el usuario, (c) la direccionalidad y (d) la función (cf. KROMANN; RIIBER; ROSBACH, 1991; CARVALHO, 2001, p. 47-58; WELKER, 2004, p. 193-214; BUGUEÑO; DAMIM, 2005).

Respecto al usuario se debe distinguir entre los hablantes de la lengua $\mathrm{A}$ y los hablantes de la lengua $\mathrm{B}$, para definir claramente a cuál de los dos públicos el diccionario estará destinado. La direccionalidad está relacionada con el hecho de que la lengua materna del usuario se toma como lengua de partida o lengua de llegada. La función se define por el tipo de tarea que el usuario va a realizar. De esta manera, el diccionario puede ser activo (para la codificación en la lengua extranjera) o pasivo (para la decodificación de la lengua extranjera). Tomando en consideración esas tres primeras variables, tendríamos para cada par de lenguas (en nuestro caso, el portugués y el español) por lo menos cuatro diferentes diccionarios:

1) Para hablantes nativos del portugués:

Diccionario I: español-portugués para decodificación (pasivo)

Diccionario II: portugués-español para codificación (activo)

2) Para hablantes nativos del español:

Diccionario I’: portugués-español para decodificación (pasivo)

Diccionario II': español-portugués para codificación (activo) 
Considerando específicamente la relación entre el español y el portugués, es necesario tratar dos cuestiones fundamentales: (a) el anisomorfismo entre las lenguas y (b) el perfil del usuario.?

El anisomorfismo, o sea, el hecho de que no hay correspondencia directa entre formas y estructuras de dos lenguas (cf. ZGUSTA, 1971, p. 294-297; MERZAGORA, 1987, p. 98-99; HARTMANN, 1991, p. 2856-2858; SZENDE, 1996; HARTMANN; JAMES, 2001, s.v. anisomorphism), se torna más evidente entre lenguas que presentan divergencias tipológicas (respecto a sus rasgos característicos) y genéticas (respecto al parentesco etimológico), como es el caso del español y del alemán:

Ejemplo: español comer $\rightarrow$ alemán essen (para seres humanos) fressen (para animales)

Sin embargo, las divergencias también se pueden encontrar en el contraste entre lenguas con una mayor convergencia tipológica y genética, como el español y el portugués:

Ejemplo: portugués aniversário $\rightarrow$ español aniversario (de un hecho) cumpleaños (de una persona)

En el nivel léxico, mencionamos también los realia, que constituyen la forma más radical de manifestación del anisomorfismo entre dos lenguas. Los realia son términos de una lengua que designan un hecho particular de la cultura de un pueblo (cf. DUBOIS et al., 1999, s.v. realia). ${ }^{10}$ Ponemos como ejemplos de realia en el portugués unidades léxicas como sarapatel "plato típico del nordeste de Brasil, hecho a base de menudos de cerdo" y micareta "fiesta de carnaval en el nordeste de Brasil, que acontece fuera del periodo anterior a cuaresma", y en el español, cueca "danza nacional de Chile" y guacamole "plato típico de México, hecho a base de aguacate". ${ }^{11}$

\footnotetext{
${ }^{9}$ Las consideraciones que serán hechas a continuación sirven también para la concepción de un diccionario monolingüe para aprendices.

${ }^{10}$ En Zgusta (1971), este fenómeno se denomina "culture-bound words".

${ }^{11}$ Aunque el léxico es el nivel donde el anisomorfismo se torna más evidente, también es posible encontrar ejemplos de este fenómeno en los niveles fonológico, morfológico y sintáctico, como en el ejemplo: portugués: gostar (de alguém/algo)/español: gustarle (algo/alguien a alguien).
} 
Respecto al perfil del usuario, si los estudiantes brasileños constituyen el público destinatario de un diccionario bilingüe, entonces la obra debe estar pensada para atender a las necesidades específicas de ese grupo de usuarios. Así, pues, en el momento de la concepción de la obra, el lexicográfo deve darle especial atención a las dificultades que los aprendices cuya lengua materna es el portugués van a encontrar, especialmente a consecuencia del fenómeno del anisomorfismo. A continuación listamos algunos de los problemas lingüísticos más comunes de los brasileños aprendices de español (en especial en los niveles iniciales):

1) Respecto al significado:

- falsos amigos.

2) Respecto a las divergencias gramaticales:

- uso de la preposición a delante de objetos directos que designan a seres humanos.

- cálculo del uso del complemento dativo.

- uso del pronombre personal átono reduplicando el complemento dativo.

- verbos que exigen complemento distinto al que se usa en portugués (por ejemplo, gustar).

- régimen preposicional distinto al portugués.

Teniendo en consideración las cuestiones involucradas en el contraste entre dos lenguas que discutimos, expondremos a continuación algunos problemas encontrados en los diccionarios bilingües español-portugués/ portugués-español. Empezamos por los problemas con las equivalencias. Proponemos el análisis de un artículo de DMiEP (1999) en la dirección portugués-español:

almofada [...] $s f$ almohada, alcatifa, almohadón; cojinete. almofada de tinta para carimbo tampón. (DMiEP, 1999, s.v. almofada)

Suponiendo que el estudiante brasileño se acerque al diccionario con el objetivo de encontrar un equivalente en español para el portugués almofada, la probabilidad de que la consulta resulte exitosa es mínima. Hay que considerar, en primer lugar, que en la variedad brasileña del portugués existen solamente dos acepciones corrientes de almofada: "saco relleno de materia esponjosa" y "almohadilla para mojar los timbres", de manera que habría que considerar como equivalentes solamente estas dos significaciones. Para la 
primera acepción, el diccionario podría ofrecer, como de hecho lo hizo, los equivalentes almohadón y almohada. ${ }^{12}$ No se entiende bien, sin embargo, porqué es que el diccionario ofreció los equivalentes alcatifa, que según el DUE (2001), además de antiguo, ${ }^{13}$ significa "alfombra" y cojinete, que tampoco consituye una equivalencia del portugués almofada (cf. DRAE, 2001; DUE, 2001), $y$, al igual que alcatifa, parece estar desusado. ${ }^{14}$ Respecto a la segunda acepción de almofada, DMiEP (1999, s.v.) la registra como si fuera una expresión fija, lo que no corresponde a la realidad del hecho lingüístico. ${ }^{15}$ Lo correcto sería presentar solamente el equivalente tampón acompañado de un especificador semántico. Por fín, sería importante que el diccionario presentara un número delante de las diferentes acepciones, para ayudar el usuario a distinguirlas.

Analicemos aún otro artículo sacado del mismo diccionario:

bajulador [...] adj halagador, servil; zalamero; lameculos. Fam. pelotilla. Fig. camaleón. (DMiEP, 1999, s.v. bajulador)

Entre tantas opciones de equivalentes para recubrir el contenido semántico de bajulador en portugués “[persona] que adula a [alguien]”, el usuario tendrá dificultades para elegir la más apropiada. Además de eso, los equivalentes servil y zalamero no corresponden exactamente al contenido

\footnotetext{
${ }^{12}$ Señalamos, sin embargo, que el español almohada es nada más que un equivalente parcial del portugués almofada, como intentamos representar en el esquema abajo: español almohada $\rightarrow$ portugués almofada travesseiro

${ }^{13}$ En el Corpus de Referencia del Español Actual (CREA), encontramos solamente dos casos de esta palabra, el primero en Costa Rica y el segundo en Cuba, ambos del año 1992.

${ }^{14}$ En el CREA, encontramos solamente seis casos de esta palabra, de los años 1975, 1992, 1996 (dos veces), 1998 y 2004.

${ }^{15}$ En la ausencia de un corpus de referencia para el portugués brasileño, no nos queda otra opción que efectuar nuestras consultas en herramientas de búsqueda disponibles en la internet, como es el caso del Google, aunque tenemos consciencia de las limitaciones de ese instrumento (cf. KILGARRIFF; GREFENSTETTE, 2008). En una consulta realizada en el Google en 07/10/2008, encontramos solamente 4 casos de la expresión almofada para tinta de carimbo, lo que nos hace creer que de hecho esa no es una estructura fija del portugués.
} 
semántico del portugués bajulador, de modo que el aprendiz de español podrá equivocarse al utilizarlas. Más grave aún son los casos de los equivalentes pelotilla y camaleón, ambas completamente equivocadas. La primera designa la "acción de adular" y no se aplica con función adjetival a la "[persona] que adula". La segunda, a su vez, designa el contenido semántico "[alguien] que es disimulado". Por otra parte, la equivalencia lameculos, aunque de hecho recubre el contenido semántico de bajulador, no es una designación neutra, a pesar de que DMiEP (1999, s.v. bajulador) no lo informa: De acuerdo con el DRAE (2001, s.v. lameculos) está marcado como "vulgar", y de acuerdo con el DUE (2001, s.v. lameculos) es "grosero". Ese hecho la convierte en una información poco funcional para un aprendiz que necesita usar el equivalente en un contexto neutro. Al fin y al cabo, el único equivalente útil para el estudiante es halagador. De igual manera lo sería adulador, que el diccionario no presenta.

Analicemos también un artículo de DSEP (2003) en la dirección español-portugués:

mamón $m$. Bot. 1. Árvore que produz o mamão. Mamoeiro. 2. Esse fruto. Mamão. 3. (Arg.) Ver borracho. Bêbado. (DSEP, 2003, s.v. mamón)

Pongamos especial atención a las acepciones 1 y 2 . En estas situaciones, DSEP (2003, s.v. mamón) presenta equivalentes completamente equivocados. El español mamón es la designación para un "fruto pequeño y de color verde, que contiene un hueso con una almendra”, mientras el portugués mamão es la designación para un "fruto grande de forma ovalada con pulpa blanda de color anaranjado y pepitas color negro en su interior". Así, pues, al contrario de lo que las informaciones presentadas nos hacen creer, el español mamón y el portugués mamão son falsos amigos, hecho que el diccionario debería poner en relieve. Además de eso, mamón es un realia. Este es más un hecho al cual DSEP (2003) tendría que haberle puesto atención, ya que si no hay cómo ofrecer un equivalente en la lengua de destino, el diccionario tiene que proporcionarle al usuario una paráfrasis que le permita entender el significado de la unidad léxica de la lengua extrajera.

Pasemos a examinar brevemente la presentación de las informaciones gramaticales. Transcribimos abajo un artículo de DCEP (2004) en la dirección portugués-español: 
gostar vi: de gustar a algn; (férias, viagem etc) disfrutar; -se vpr gustarse; - de fazer aco gustarle a algn hacer algo; não gostei do filme no me gustó la película; eu ia de ir me gustaría ir; mais de... preferir... (DCEP, 2004, s.v. gostar)

Desde el punto de vista del estudiante brasileño, el artículo consultado debería servir para la función activa. Así, pues, el diccionario debería presentar la información sobre la construcción sintáctica del verbo gustar (que, en este caso, es el equivalente) de forma clara para el usuario, buscando enfatizar la diferencia que hay entre el español y el portugués. Sin embargo, DCEP (2004, s.v. gostar) ofrece una serie de informaciones poco útiles para el estudiante brasileño y, principalmente, de manera no muy clara.

\section{Learner's dictionaries}

El learner's dictionary, o diccionario para aprendices, se define como "a pedagogical dictionary aimed primarily at non-native learners of a language" (HARTMANN; JAMES, 2001, s.v. learner's dictionary). Si la concepción de los learner's dictionaries tuviera en cuenta un público-destinatario hablante nativo de una lengua determinada, los principios del anisomorfismo lingüístico y de la definición del perfil del usuario, que discutimos en el apartado anterior, deberían estar plenamente aplicados. No obstante, y lamentablemente, esto no suele ocurrir. ${ }^{16}$ DSLE (1996), siguiendo el ejemplo de diccionarios de otras tradiciones lexicográficas, como CCLD (2003), OALD (2005) y LGDaF (2008), ha sido concebido para atender a las necesidades de los estudiantes de español como lengua extranjera en general, sin considerar que las dificultades con las que un aprendiz brasileño de español se enfrenta son muy distintas de las de un aprendiz danés, por exemplo. Por otra parte, el DELE (2002) ha sido presentado al público brasileño como una obra proyectada especialmente para atender a sus necesidades. Esta obra, sin embargo, reproduce integralmente el contenido del Diccionario Vox para la Enseñanza de la Lengua Española, de modo que, salvo por el hecho de ofrecer equivalentes en portugués, no hay nada en DELE (2002) que demuestre una preocupación efectiva con el aprendiz brasileño.

Considerando que los aprendices tienen que cumplir tareas de comprensión y producción lingüísticas, evaluaremos la utilidad de los learner's

${ }^{16}$ Eso se puede comprobar igualmente mediante el análisis de los learner's dictionaries del inglés y del alemán (cf. BUGUEÑO; JARDIM, 2010). 
dictionaries como auxiliares en estas actividades. Así, pues, analizaremos, en primer lugar, la calidad de las paráfrasis definitorias, y en segundo lugar, la presentación de las informaciones gramaticales, en especial las sintácticas.

La paráfrasis definitoria es el segmento más importante del artículo léxico de un diccionario semasiológico, $y$, a la vez, el que más plantea problemas para el lexicógrafo. Aunque hayan sido publicados innumerables trabajos sobre el tema de la definición lexicográfica (cf., por ejemplo, POTTIER, 1977; REY, 1977, p. 98-113; BOSQUE, 1982; WERNER, 1984; SECO, 1987, p. 15-45; MARTÍNEZ DE SOUZA, 1995, s.v. definición lingüistica; LANDAU, 2001, p. 153-216; SCHLAEFER, 2002, p. 96-99; JACKSON, 2002, p. 86-100; GEERAERTS, 2003; HANKS, 2003), según Bugueño (2009), hasta el día de hoy no tenemos estudios conclusivos sobre cómo generar una definición satisfactoria. Creemos, sin embargo, que la atención a los principios de concisión, compleción y circularidad, propuestos por Martínez de Souza (1995, s.v. definición lingüistica), al igual que la aplicación de criterios muy sencillos, como el isomorfismo morfológico, el uso de un lenguaje de fácil comprensión y el empleo de contornos semánticos en las definiciones, presentados en Beneduzi; Bugueño; Farias (2005), podrían contribuir a mejorar la calidad de las paráfrasis definitorias de un modo general. Así, pues, problemas como los siguientes podrían ser evitados:

adolescente [...] adj.-com. (persona) Que está en la adolescencia o que tiene relación con esa edad: a los adolescentes les encanta que los traten como personas adultas; tengo dos hijas adolescentes; recordó sus confidencias adolescentes con su mejor amigo. adolescente (DELE, 2002, s.v. adolescente)

compinche [...] com. fam. Amigo o compañero; especialmente, persona con la que se ha realizado una mala acción: él y sus compinches irán a la cárcel. cúmplice (DELE, 2002, s.v. compinche)

Ambas definiciones mezclan dos acepciones distintas, que deberían presentarse separadamente. Esta práctica es contraria al principio de la circularidad propuesto por Martínez de Souza (1995), considerando que las definiciones de DELE (2002) no pueden sustituir a la unidad léxica en un contexto.

El problema más grave de los learner's dictionaries de lengua española, sin embargo, está en la presentación de las informaciones sintácticas. A continuación, expondremos y analizaremos algunos casos: 
enviar [...] 2 [algo] Hacer llegar a un lugar: ¡cuándo me enviaste la postal?; nos enviaron el paquete por correo urgente. $\Leftrightarrow$ recibir. [...] enviar (DELE, 2002, s.v. enviar)

ofrecer [...] 3 [algo; a alguien] Proponer o dar oportunidad o facilidad para hacer una cosa: le han ofrecido ser director de un hotel. $\Rightarrow$ brindar, proporcionar. propor [...] (DELE, 2002, s.v. ofrecer)

Es cierto que los diccionarios de lengua española acostumbran a no indicar el dativo en los artículos. Un diccionario destinado a aprendices extranjeros, sin embargo, debería preocuparse en facilitarle al usuario el mayor número posible de informaciones que puedan ayudarlo tanto en las tareas de comprensión como en las de producción lingüística. Así, pues, se debería marcar el dativo en estas obras con el objetivo de ayudar a los usuarios a calcular el uso de ese elemento. No obstante, s.v. enviar, además de no informar que el verbo en cuestión exige dativo, DELE (2002) indica el complemento indirecto mediante el empleo de las formas pronominales sincréticas de primera persona del singular y del plural en los ejemplos, que serían el segmento donde el estudiante podría rescatar la información sintáctica. Por otra parte, s.v. ofrecer, DELE (2002) actúa irreprochablemente al señalar el dativo en el segmento reservado para la indicación sintáctica y en el ejemplo, a través del pronombre dativo de tercera persona (que, en este caso, se contrapone a los pronombres acusativos de tercera persona $l o / l a)$.

Encontramos también otros problemas respecto a la presentación de los complementos verbales en los learner's dictionaries:

disculpar $v$. tr. 1. Encontrar o presentar <una persona> una explicación que libre de una culpa o una obligación a [otra persona] o [una cosa]: El calor disculpa todo en estas fechas. Debemos disculparle, porque es despistado. Siempre se disculpan los errores de los amigos. SIN. excusar. [...] (DSLE, 1996, s.v. disculpar)

morder [...] 1 tr.-prnl. [algo, a alguien] Sujetar y apretar clavando los dientes: el niño muerde la manzana; un perro le mordió en la pierna. morder [...] (DELE, 2002, s.v. morder)

Tenemos conciencia de que ambos verbos pueden construirse con dativo (Juan le disculpó los errores al amigo, El perro le mordió la pierna al niño). No obstante, en ninguno de los artículos que transcribimos se enfatiza la construcción con el dativo. Por otro lado, el uso del leísmo en los ejemplos subrayados puede llevar al usuario a una interpretación equivocada. En una 
obra destinada a aprendices, los ejemplos leístas deberían ser evitados, ya que solamente contribuyen a confundir al estudiante que aún no consigue calcular bien el uso de los objetos directo e indirecto del español. ${ }^{17}$

\section{Diccionarios generales de lengua}

Un aprendiz de español con un buen dominio del idioma puede comenzar a usar los diccionarios generales de lengua. Teniendo en cuenta que los usuarios necesitan resolver problemas relativos a la significación de las palabras y su empleo sintáctico, al igual que en el caso anterior, evaluaremos, por un lado, la calidad de las definiciones de los diccionarios generales, y por el otro, el provecho efectivo que pueden tener las informaciones sintácticas presentes en estas obras para los estudiantes brasileños.

En lo concerniente a las paráfrasis definitorias, hay que señalar que los problemas encontrados en los diccionarios generales del español son similares a los de los learner's dictionaries. A continuación, presentamos una breve taxonomía de los vicios de las definiciones de los diccionarios generales del español:

1) Paráfrasis definitorias en las que se utilizan muchos términos técnicos:

drupa [...] 1. f. Bot. Fruto de mesocarpio carnoso y endocarpio leñoso y una sola semilla; p. ej., el melocotón y la ciruela. (DRAE, 2001, s.v. drupa)

papayo [...] m. Árbol caricáceo propio de países cálidos, coronado por grandes hojas palmeadas; tiene un látex abundante que contiene un fermento parecido a la pepsina. $\approx$ Lechoso, papayero. $\Rightarrow$ Botuto, chamburo. $\alpha *$ Planta. (DUE, 2001, s.v. papayo)

2) Paráfrasis definitorias que mezclan dos acepciones:

pinche $^{2}[\ldots]$ adjetivo $\mid$ nombre común 4 Méx coloquial despectivo [cosa] Que es sumamente desagradable, despreciable o de pésima calidad [...] (DUEA, 2000, s.v. pinche $\left.{ }^{2}\right)$

sandía [...] 1. f. Planta herbácea anual, de la familia de las Cucurbitáceas, con tallo velloso, flexible, rastrero, de tres a cuatro metros de largo, hojas

17 El panorama del leísmo está lejos de la sencillez (cf., por ejemplo, CAMPOS, 1999; DPD, 2005, s.v. leísmo). La complejidad de la cuestión es un argumento muy fuerte para rechazar los ejemplos leístas en los learner's dictionaries del español. 
partidas en segmentos redondeados y de color verde oscuro, flores amarillas, fruto casi esférico, tan grande que a veces pesa $20 \mathrm{~kg}$, de corteza verde uniforme o jaspeada y pulpa encarnada, granujienta, aguanosa y dulce, entre la que se encuentran, formando líneas concéntricas, muchas pepitas negras y aplastadas. Es planta muy cultivada en España. 2. f. Fruto de esta planta. (DRAE, 2001, s.v. sandía)

3) Paráfrasis definitorias lingüísticamente mal formuladas:

novio, -a [...] 1 n. Persona que se casa o acaba de *asarse: 'Traje de novia. Viaje de novios'. 2 Con respecto a una persona, otra que mantiene con ella relaciones amorosas con intención de casarse con ella. [...] (DUE, 2001, s.v. novio, -a)

4) Paráfrasis definitorias excesivamente largas (con dos o más periodos): ${ }^{18}$

ciruela [...] 1. f. Fruto del ciruelo. Es una drupa, muy variable en forma, color y tamaño según la variedad del árbol que la produce. El epicarpio suele separarse fácilmente del mesocarpio, que es más o menos dulce y jugoso y a veces está adherido al endocarpio. [...] (DRAE, 2001, s.v. ciruela)

5) Paráfrasis definitorias sinonímicas (círculo vicioso en la definición):

gestante [...] 1. adj. Que gesta. 2. adj. embarazada. U. t. c. s. f. (DRAE, 2001, s.v. gestante)

embarazado, da [...] 1. adj. Dicho de una mujer: preñada. U. t. c. s. f. (DRAE, 2001, s.v. embarazado, da)

encinta [...] 1. adj. Dicho de una mujer: preñada. (DRAE, 2001, s.v. encinta)

preñado $^{2}$, da [...] 1. adj. Dicho de una mujer, o de una hembra de cualquier especie: Que ha concebido y tiene el feto o la criatura en el vientre. [...] (DRAE, 2001, s.v. preñado², da)

Como ya hemos dicho, una solución definitiva para la generación de buenas definiciones todavía no la tenemos. Sin embargo, si se hubieran seguido criterios tan básicos como los expuestos en el apartado anterior, equívocos como los que vemos en esas definiciones podrían haber sido evitados.

${ }^{18}$ La definición de sandía del DRAE (2001) también serviría para ilustrar este problema. 
Nos queda aún por analizar las informaciones sintácticas de los diccionarios generales de lengua española. En este ámbito, destacamos el DUE (2001), por lo que dijimos anteriormente acerca de la presentación de los regímenes preposicionales y de las colocaciones, pero también por la indicación de los actantes en las paráfrasis definitorias, como en el ejemplo ofrecido a continuación:

comprar [...] 1 («a, de, en, por») tr. o abs. *Adquirir [una cosa] mediante dinero: 'Ha comprado una finca. Comprar a los vendedores ambulantes [directamente del productor, en la tienda de la esquina, por metros, por litros]'. $\odot$ El complemento de persona puede ser la persona de quien se adquiere y aquella para quien se adquiere; si existen los dos, el segundo va con "para»: 'Le compré su pluma. Les compra todo lo que le piden. Le compró al viajante un abrigo para su mujer'. [...] (DUE, 2001, s.v. comprar)

No obstante, respecto a la indicación del dativo, el DUE (2001), al igual que las demás obras de lengua española, sigue siendo poco sistemático:

enviar [...] 1 tr. Hacer que [una cosa] *llegue a cierto sitio o a alguien que está a cierta distancia: 'El gobierno ha enviado socorros a la zona damnificada. Le ha enviado un ramo de flores'. [...] (DUE, 2001, s.v. enviar)

enviar [...] 2. tr. Hacer que algo se dirija o sea llevado a alguna parte. [...] (DRAE, 2001, s.v. enviar)

El DUE (2001, s.v. enviar) no presenta ninguna indicación explícita del uso del dativo, de modo que el usuario solamente puede rescatar esta información a través de los ejemplos. En peor situación, sin embargo, está el DRAE (2001, s.v. enviar), que simplemente omite la información sobre el dativo.

Uno de los grandes problemas que el estudiante brasileño seguirá encontrando en algunas de estas obras es el empleo del leísmo en los ejemplos, como vemos a continuación:

ayudar [...] v.tr. 1 Prestar cooperación en una actividad en la que corresponde a otro la responsabilidad o el esfuerzo mayor: les ayudó a aprobar el examen de ingreso. [...] (GDLE, 2001, s.v.)

reprochar [...] tr. Dirigir a [alguien] *quejas o censuras por [algo] que ha hecho o dicho, por sus sentimientos, etc.; el complemento directo 
puede ser la persona o la cosa: 'Le reprochan por su egoísmo. Le reprochan su egoísmo. Se reprocha a sí mismo su imprevisión. No tienes nada que reprocharte'. [...] (DUE, 2001, s.v.)

Así, pues, el estudiante brasileño de español que consulta una obra como el DUE (2001) debe poner atención a este hecho para evitarse problemas que puedan generarse de malas interpretaciones. Dicho en otros términos, es el dominio del idioma lo que le va a garantizar al aprendiz el éxito de la consulta en estos casos.

\section{Consideraciones finales}

El análisis realizado y presentado sintéticamente en este trabajo nos permitió constatar:

1) Respecto a los diccionarios bilingües, aunque los estudiantes brasileños cuentan con una enorme cantidad de esas obras, la mayoría, a ejemplo de las que analizamos, sirven de forma muy precaria a la función de comprensión y simplemente no son útiles para la función de producción lingüística ${ }^{19}$. Esto se explica por el hecho de que la concepción de los diccionarios bilingües utilizados en Brasil ignora completamente principios fundamentales como la distinción entre pasivo/activo, el anisomorfismo lingüístico y la definición de un perfil de usuario.

2) Respecto a los learner's dictionaries, hace falta señalar, en primer lugar, que no es mucha la variedad de obras de este tipo para la lengua española con las que el estudiante brasileño cuenta. Además de eso, los problemas conceptuales de esas obras tampoco les permiten cumplir satisfactoriamente con su función de auxiliar en el proceso de enseñanza del español como lengua extrajera. En este ámbito, se echa de menos una obra concebida especialmente para atender a las necesidades de los aprendices brasileños de español.

3) Por fin, respecto a los diccionarios generales del español, hay que tener en cuenta dos factores. En primer lugar, aunque estos diccionarios son, de hecho, las mejores herramientas con las que el estudiante brasileño puede

${ }^{19}$ Para un análisis de los diccionarios bilingües español-portugués/portugués-español, cf. también Farias (2009). 
contar, todavía es posible mejorarlos cambiando algunas paráfrasis definitorias o perfeccionando el sistema de indicaciones sintácticas. En segundo lugar, para un real aprovechamiento de todas las informaciones presentes en los diccionarios de lengua española, hace falta que el estudiante tenga no solamente una muy buena competencia en la lengua extranjera, sino que también sea entrenado en el uso de los diccionarios, lo que, lamentablemente, no es algo tan obvio en nuestro medio.

\section{Referencias}

ALVAR EZQUERRA, M. Lexicografía. In: HOLTUS, G.; METZELTIN, M.; SCHMITT, C. (Hrsg.). Lexikon der Romanistischen Linguistik. Tübingen: Niemeyer, 1992. p. 636-649.

BENEDUZI, R.; BUGUEÑO, F.; FARIAS, V. Avanços na redação de um dicionário de falsos amigos espanhol-português. Lusorama, Frankfurt am Main, v. 61/62, p. 195-219, 2005.

BOSQUE, I. Sobre la teoría de la definición lexicográfica. Verba, Santiago de Compostela, v. 9, p. 105-123, 1982.

BUGUEÑO, F. Cómo leer y qué esperar de un diccionario monolingüe (con especial atención a los diccionarios del español). Revista Língua \& Literatura, Frederico Westphalen, v. 8-9, p. 97-114, 2003.

BUGUEÑO, F. Reseña a: Real Academia Española. Diccionario panhispánico de dudas. Madrid: Santillana, 2005. Anuario Brasileño de Estudios Hispánicos, São Paulo, n. 16, p. 187-189, 2006a.

BUGUEÑO, F. Léxico e ensino: Señas (2000), um dicionário para aprendizes do espanhol? In: MARTINS, E. S.; CANO, W. M.; MORAES FILHO, W. B. (Orgs.). Léxico e morfofonologia: perspectivas e análises. Uberlândia: EDUFU, 2006b. p. 213-227.

BUGUEÑO, F. Panorama da lexicografia alemã. Contingentia, Porto Alegre, v. 3, n. 2, p. 89-110, 2008. Disponible en: <http://www.seer.ufrgs.br/ index.php/contingentia/article/view/-6508/4241 >. Acceso en: 30 Dic. 2008.

BUGUEÑO, F. Para uma taxonomia de paráfrases explanatórias. Alfa, São Paulo, v. 53, n. 1, p. 243-260, 2009.

BUGUEÑO, F.; DAMIM, C. P. Elementos para uma escolha fundamentada de dicionários bilíngües português/inglês. Entrelinhas, São Leopoldo, v. 3, p. 1-9, 2005. Disponible en: <http://www.entrelinhas.unisinos.br/_include/imprimir_artigo. inc.php?e=3\&a=18>. Acceso en: 07 Ene. 2008. 
BUGUEÑO, F.; FARIAS, V. S. Desenho da macroestrutura de um dicionário escolar de língua portuguesa. In: XATARA, C.; BEVILACQUA, C. R.; HUMBLÉ, Ph. (Orgs.). Lexicografia Pedagógica: Pesquisas e perspectivas. Florianópolis: UFSC/NUT, 2008. p. 129-167. Disponible en: <http:// www.cilp.ufsc.br/LEXICOPED.pdf>. Acceso en: 30 Abr. 2009.

BUGUEÑO, F.; FARIAS, V. S. Panorama crítico dos dicionários escolares brasileiros. Lusorama, Frankfurt am Main, v. 77/78, p. 29-78, 2009.

BUGUEÑO, F.; JARDIM, C. R. Os learner's dictionaries do inglês e os Lernwörterbücher do alemão: uma simples transposição de nomes? Contingentia, Porto Alegre, v. 5, n. 1, p. 41-67, 2010. Disponible en: <http://www.seer.ufrgs.br/ index.php/contingentia/article/view/13319/7614>. Acceso en: 23 Jul. 2010.

CAMPOS, H. Transitividad e intransitividad. In: BOSQUE, I.; DEMONTE, V. (Orgs.). Gramática descriptiva de la lengua española. Madrid: Espasa-Calpe, 1999. p. 1519-1574.

CARVALHO, O. L. Lexicografia bilíngüe português/alemão: Teoria e aplicação à categoria das preposições. Brasília: Thesaurus, 2001.

CASARES, J. Diccionario ideológico de la lengua española. Barcelona: Editorial Gustavo Gili, 2007.

CASTILLO, A.; GARCÍA, J. M. La lexicografía didáctica. In: MEDINA GUERRA, A. (Org.). Lexicografía Española. Barcelona: Ariel, 2003. p. 333-351. CCLD. HARPER COLLINS. Collins Cobuild Advanced Learner's Dictionary. Glasgow: Lingea Lexicon, 2003.

CREA. REAL ACADEMIA ESPAÑOLA. Banco de datos. Corpus de referencia del español actual. Disponible en: <http://www.rae.es>. Acceso en: 25 Sept. 2008.

DCEP. HARPER COLLINS. Dicionário Collins: espanhol-português/ português-espanhol. São Paulo: Martins Fontes, 2004.

DELE. UNIVERSIDAD DE ALCALÁ DE HENARES. Señas: Diccionario para la enseñanza de español para brasileños. São Paulo: Martins Fontes, 2002.

DERAE. REAL ACADEMIA ESPAÑOLA. Diccionario del estudiante. Madrid: Santillana, 2005.

DMiEP. PEREIRA, H.; SIGNER, R. Dicionário Michaelis espanhol-português/ português-espanhol. São Paulo: Melhoramentos/Publifolha, 1999.

DPD. REAL ACADEMIA ESPAÑOLA. Diccionario panhispánico de dudas. Madrid: Santillana, 2005.

DPRAE. REAL ACADEMIA ESPAÑOLA. Diccionario práctico del estudiante. Madrid: Santillana, 2007. 
DRAE. REAL ACADEMIA ESPAÑOLA. Diccionario de la lengua española. Madrid: Espasa-Calpe, 2001.

DSAEC. ESPASA-CALPE. Diccionario de sinónimos y antónimos. Madrid: Espasa-Calpe, 1997.

DSALE. CORRIPIO, F. Diccionario de sinónimos y antónimos de la lengua española. Barcelona: Larousse Editorial, 1998.

DSAV. VOX. Diccionario avanzado de sinónimos y antónimos de la lengua española. Barcelona: Bibliograf, 1998.

DSEP. DIAZ Y GARCÍA-TALAVERA, M. Dicionário Santillana para estudantes: espanhol-português/português-espanhol. São Paulo: Moderna, 2003.

DSLE. UNIVERSIDAD DE SALAMANCA. Diccionario Salamanca de la lengua española. Madrid: Santillana, 1996.

DUBOIS, J. et al. Dictionnaire de linguistique et des sciences du langage. Paris: Larousse, 1999.

DUE. MOLINER, M. Diccionario de uso del español. Madrid: Gredos, 2001.

DUEA. VOX. Diccionario de uso del español de América y España. Barcelona: Spes Editorial, 2000.

ENGELBERG, S.; LEMNITZER, L. Lexikographie und Wörterbuchbenutzung. Tübingen: Stauffenburg, 2004.

EvAm. EVEREST. Diccionario práctico de Americanismos. León: Everest, 1987.

FARIAS, V. S. Dicionários escolares de língua portuguesa: uma breve análise de aspectos macroestruturais. Lusorama, Frankfurt am Main, v. 71/72, p. 160-206, 2007.

FARIAS, V. S. Análise de alguns aspectos microestruturais em quatro dicionários bilíngües espanhol-português/português-espanhol. Anuario Brasileño de Estudios Hispánicos, São Paulo, n. 19, p. 175-192, 2009.

FORNARI, M. K. A problemática dos americanismos: teorias de divisão dialetal, análise de dados e uma proposta para a marcação diatópica do Dicionário de Falsos Amigos Espanhol-Português. Revista de Letras, Curitiba, v. 70, p. 223-246, 2006. GDEA. SOCIEDAD GENERAL ESPAÑOLA DE LIBRERÍA. Gran diccionario de uso del español actual. Madrid: Alcobendas, 2001.

GDLE. LAROUSSE. Gran Diccionario de la Lengua Española. Barcelona: Spes Editorial, 2001.

GEERAERTS, D. Meaning and definition. In: STERKENBURG, P. (Org.). A practical guide to lexicography. Amsterdam/Philadelphia: John Benjamin, 2003. p. 83-93. 
HAENSCH, G. et al. La lexicografía: de la lingüística teórica a la lexicografía práctica. Madrid: Gredos, 1982.

HANKS, P. Definitions and Explanations. In: HARTMANN, R. R. K. (Ed.). Lexicography: Critical concepts III. London/New York: Routledge, 2003. p. 191206

HARTMANN, R.R.K. Contrastive Linguistics and Bilingual Lexicography. In: HAUSMANN, F. J. et al. (Hrsgn.). Wörterbücher, dictionaries, dictionnaires. Ein internationales Handbuch zur Lexikographie. Berlin/New York: Walter de Gruyter, 1991. p. 2854-2859.

HARTMANN, R.R.K. Teaching and researching lexicography. London: Longman, 2001.

HARTMANN, R.R.K; JAMES, G. Dictionary of lexicography. London: Routledge, 2001.

HAUSMANN, F. J. Wörterbuchtypologie. In: HAUSMANN, F. J. et al. (Hrsgn.). Wörterbücher, dictionaries, dictionnaires. Ein internationales Handbuch zur Lexikographie. Berlin/New York: Walter de Gruyter, 1989. p. 968-988.

HERNÁNDEZ, H. La lexicografía didáctica del español: aspectos históricos y críticos. In: FUENTES MORÁN, M. T.; WERNER, R. (Eds.), Lexicografías iberorrománicas: Problemas, propuestas y proyecto. Frankfurt: Vervuert, 1998. p. $49-79$.

JACKSON, H. Lexicography. London: Routledge, 2002.

KILGARRIFF, A.; GREFENSTETTE, G. Introduction to the Special Issue on the Web as Corpus. In: FONTENELLE, T. (Ed.). Practical Lexicography: a reader. Oxford: OUP, 2008. p. 89-101

KROMANN, H., RIIBER, T.; ROSBACH, P. Principles of Bilingual Lexicography. In: HAUSMANN, F. J. et al. (Hrsgn.). Wörterbücher, dictionaries, dictionnaires. Ein internationales Handbuch zur Lexikographie. Berlin/New York: Walter de Gruyter, 1991.p. 2711-2728.

KÜHN, P. Typologie der Wörterbücher nach Benutzungsmöglichkeiten. In: HAUSMANN, F. J. et al. (Hrsgn.). Wörterbücher, dictionaries, dictionnaires. Ein internationales Handbuch zur Lexikographie. Berlin/New York: Walter de Gruyter, 1989. p. 111-127.

LANDAU, S. Dictionaries: The art and craft of lexicography. Cambridge: CUP, 2001.

LEA. LONGMAN GROUP LIMITED. Longman Essential Activator. Harlow/ Essex: Longman, 2006. 
LGDaF. GÖTZ, D., HAENSCH, G.; WELLMANN, H. Großwörterbuch Deutsch als Fremdsprache. Berlin/München: Langenscheidt, 2008.

MARTÍN MINGORANCE, L. La lexicografía onomasiológica. In: HERNÁNDEZ, H. (Coord.). Aspectos de lexicografía contemporánea. Barcelona: Bibliograf, 1994. MARTÍNEZ DE SOUZA, J. Diccionario de lexicografía práctica. Barcelona: Bibliograf, 1995.

MERZAGORA, G. M. La lessicografia. Bologna: Zanichelli, 1987.

Mo. MORÍNIGO, M. A. Diccionario del español de América. Madrid: Anaya \& Mario Muchnik, 1996.

OALD. HORNBY, A. S. Oxford Advanced Learner's Dictionary. Oxford: OUP, 2005.

PÉREZ LAGOS, M. F. Los diccionarios escolares de los últimos años: ¿̨una nueva lexicografía didáctica? In: ALVAR EZQUERRA, M.; CORPAS PASTOR, G. (Coords.). Diccionarios, frases, palabras. Málaga: Servicio de Publicaciones de la Universidad de Málaga, 1998. p. 115-125.

PÉREZ LAGOS, M. F. A propósito del Diccionario Ideológico de la Lengua Española VOX. Su didactismo. In: AYALA CASTRO, M. C. (Coord.). Diccionarios y enseñanza. Alacalá de Henares: Servicio de Publicaciones de la Universidad de Alcalá de Henares, 2001. p. 179-192.

POTTIER, B. A definição semântica nos dicionários. Tradução Maria Angela Botelho Pereira. In: LOBATO, L. M. P. (Org.). A semântica na lingüistica moderna: o léxico. Rio de Janeiro: Francisco Alves, 1977. p. 21-31

RABANALES, A. ¿Qué es hablar correctamente? Revista de Educación, Santiago de Chile, v. 119, p. 49-58, 1984.

REY, A. Le lexique: images et modèles. Du dictionnaire à la lexicologie. Paris: Armand Colin, 1977.

SCHLAEFER, M. Lexikologie und Lexikographie. Eine Einführung am Beispiel deutscher Wörterbücher. Berlin: Erich Schmidt Verlag, 2002.

SECO, M. Estudios de lexicografía española. Madrid: Paraninfo, 1987.

SoAm. SOPENA, R. Diccionario ilustrado Sopena. Barcelona: Ramón Sopena, 1983.

SWANEPOEL, P. Dictionary typologies: A pragmatic approach. In: (Ed.). A practical guide to lexicography. Amsterdam/Philadelphia: John Benjamin, 2003. p. 44-69.

SZENDE, T. Problèmes d'équivalence dans les dictionnaires bilingues. In: BÉJOINT, H.; THOIRON, P. (Orgs.). Les dictionnaires bilingues. Louvain-laNouve: Ducolot, 1996. p. 111-126. 
WELKER, H. A. Dicionários: Uma pequena introdução à lexicografia. Brasília: Thesaurus, 2004.

WERNER, R. Semasiologische und enzyklopädische Definition im Wörterbuch. In: GOETZ, D.; HERBST, T. (Hrsgn.). Theoretische und praktische Probleme der Lexikographie. München: Max Hueber, 1984. p. 382-407.

ZANATTA, F. Análise de dicionários de uso do espanhol e do português. $82 \mathrm{f}$. Trabalho de Conclusão de Curso (Licenciatura em Letras) - Instituto de Letras, Universidade Federal do Rio Grande do Sul, Porto Alegre, 2006a. Disponible en: <www6.ufrgs.br/termisul/biblioteca/TCCZanatta.pdf>. Acceso en: 26 Oct. 2008.

ZANATTA, F. La calidad de las definiciones lexicográficas de cuatro diccionarios de americanismos. Anuario Brasileño de Estudios Hispánicos, São Paulo, n. 16, p. 135-152, 2006b.

ZGUSTA, L. Manual of Lexicography. Prague: Academia, 1971.

Recebido em 17 de março de 2010. Aprovado em 21 de julho de 2010. 